\title{
BMJ Open Clot regression effects of rivaroxaban in the treatment of venous thromboembolism in patients with cancer (CRERIT-VTE cancer): study protocol
}

\author{
Shigeki Takai, ${ }^{1}$ Naohiko Nakanishi, ${ }^{1}$ Isao Yokota, ${ }^{2}$ Kojiro Imai, ${ }^{3}$ Ayumu Yamada, ${ }^{4}$ \\ Takanori Kawasaki, ${ }^{1}$ Takashi Okada, ${ }^{5}$ Takahisa Sawada, ${ }^{6}$ Hiroshi Fujita, ${ }^{7}$ \\ Satoaki Matoba ${ }^{1}$
}

To cite: Takai S, Nakanishi N, Yokota I, et al. Clot regression effects of rivaroxaban in the treatment of venous thromboembolism in patients with cancer (CRERIT-VTE cancer): study protocol. BMJ Open 2019;9:e031698. doi:10.1136/ bmjopen-2019-031698

- Prepublication history for this paper is available online. To view these files, please visit the journal online (http://dx.doi. org/10.1136/bmjopen-2019031698).

Received 15 May 2019 Revised 12 October 2019 Accepted 16 October 2019

Check for updates

(C) Author(s) (or their employer(s)) 2019. Re-use permitted under CC BY-NC. No commercial re-use. See rights and permissions. Published by BMJ.

For numbered affiliations see end of article.

Correspondence to Dr Naohiko Nakanishi; naka-nao@koto.kpu-m.ac.jp

\section{ABSTRACT}

Introduction Anticoagulant therapy in patients with cancer with venous thromboembolism (VTE) increases the risk of both VTE recurrence and haemorrhagic complication. Direct oral anticoagulants (DOACs) have been shown to be effective in preventing VTE recurrence, and comparable to conventional therapy in preventing VTE recurrence in patients with advanced cancer. Rivaroxaban is a DOAC that causes thrombus regression, possibly through a profibrinolytic effect. Thrombus regression with initial treatment is essential for VTE patients. However, the thrombolytic effect of DOAC for VTE patients with cancer has not been fully examined. Therefore, in this study, we investigate the thrombolytic effect of rivaroxaban in patients with cancer who develop VTE. Methods and analysis This study is a single-arm, openlabel, prospective interventional study. Forty patients aged from 20 to 75 years old at the time of consent who have been diagnosed with acute VTE and have active cancer are included. Patients are excluded if they have received thrombolytic therapy, have creatinine clearance of less than $30 \mathrm{~mL} / \mathrm{min}$, have expected a life expectancy of less than 6 months or have deep vein thrombosis limited to the distal lower leg. Eligible patients receive standard treatment with rivaroxaban ( $15 \mathrm{mg}$ two times daily for 3 weeks, followed by $15 \mathrm{mg} \mathrm{QD}$ ). The primary study endpoint is clot regression ratio as evaluated by contrast-enhanced CT imaging. CT imaging is obtained at baseline, $21 \pm 4$ and $90 \pm 14$ days after the start of rivaroxaban treatment. Secondary endpoints are the recurrence of VTE and haemorrhagic complications.

Ethics and dissemination This study was approved by the institutional review board of the Kyoto Prefectural University of Medicine. Study results will be disseminated through peerreviewed journals.

Trial registration number

UMIN000027793

\section{INTRODUCTION}

In patients who developed acute venous thromboembolism (VTE), such as deep vein
Strengths and limitations of this study

- This study is the first prospective interventional study in Japan to assess the clot regression of rivaroxaban in patients with cancer who develop venous thromboembolism (VTE).

- This study will answer the clinical question whether the Japanese dose of rivaroxaban ( $15 \mathrm{mg}$ two times daily for 3 weeks, followed by $15 \mathrm{mg} Q D$ ) is effective and safe for the VTE patients with cancer.

- In this exploratory study, we could investigate thrombolytic effect of rivaroxaban in patients with cancer who develop VTE. In a small number of patients, we might not be able to evaluate secondary endpoints such as recurrence of symptomatic VTE or haemorrhagic complications.

thrombosis or pulmonary embolism (PE), about $10 \%-20 \%$ are complicated by anamnestic or active cancer. ${ }^{1-3}$ In patients with cancer with VTE, anticoagulant therapy increases the risk of both VTE recurrence and haemorrhage. Previous studies have reported that low-molecular-weight heparin (LMWH) reduced VTE recurrence without increasing the risk of haemorrhage in patients with cancer compared with vitamin K antagonists, ${ }^{4}$ substantiating LMWH as a recommended monotherapy for VTE. ${ }^{5}$

In the recent years, direct oral anticoagulants (DOACs) have also been shown to be effective in preventing VTE recurrence. In the EINSTEIN-DVT $^{6}$ and EINSTEIN-PE ${ }^{7}$ studies in which rivaroxaban, a Factor Xa inhibitor, was compared with LMWH plus warfarin for VTE recurrence, rivaroxaban was noninferior to combination therapy with enoxaparin and warfarin for VTE recurrence and superior for haemorrhagic complications. 
In a subgroup analysis of these studies, rivaroxaban was comparable in reducing VTE recurrence and led to fewer events of haemorrhage in comparison to combination therapy with enoxaparin plus warfarin in patients with cancer at a high risk of thrombosis and haemorrhage. ${ }^{8}$ Although rivaroxaban was shown to be effective and safe in this population, its utility in reducing thrombosis in patients with cancer has not been fully evaluated.

Recently, it has been indicated in prospective studies that some DOACs have efficacy and safety comparable to conventional LMWH therapy in preventing VTE recurrence in patients with advanced cancer. Oral edoxaban was non-inferior to subcutaneous LMWH when examining the composite outcome of recurrent VTE and major bleeding, ${ }^{9}$ while rivaroxaban was also associated with relatively low VTE recurrence but higher non-major bleeding in comparison to LMWH. ${ }^{10}$ Moreover, in the context of prophylaxis, apixaban has been reported to have a lower rate of VTE than placebo in patients with cancer who were initiating chemotherapy and had an intermediateto-high risk of VTE. ${ }^{11}$ In addition, thromboprophylaxis with rivaroxaban led to a lower incidence of VTE or death in high-risk ambulatory patients with cancer during the intervention period. ${ }^{12}$

Thrombus regression with rivaroxaban has been studied in the J-EINSTEIN study that investigated the efficacy of low dose rivaroxaban for the treatment of
VTE in Japanese patients. ${ }^{13}$ In this study, an imaging test combining ultrasound and lung imaging on day 22 after rivaroxaban administration revealed the percentage of patients with complete resolution of thrombus was $26.7 \%$ in the rivaroxaban group compared with $15.8 \%$ in the unfractionated heparin plus warfarin group. This suggests that rivaroxaban could have a profibrinolytic effect on thrombi. ${ }^{14}$ As residual thrombus in the acute phase can lead to post-thrombotic syndrome (PTS), thrombus regression with the initial treatment is important for VTE patients. However, the thrombolytic effect of DOAC for VTE patients with cancer has not been fully examined. Therefore, in this study, we investigate the thrombolytic effect of rivaroxaban in patients with cancer who develop VTE. We believe the results of this study will greatly contribute to improving prognosis and reducing symptoms in patients with cancer by examining the clot regression effects of rivaroxaban in the treatment of VTE.

\section{OBJECTIVE}

The aim of this study is to investigate the thrombolytic effect of rivaroxaban in patients with cancer who develop VTE (symptomatic and asymptomatic).

Patients aged from 20 up to 75 years old who have been diagnosed with acute VTE and have active cancer

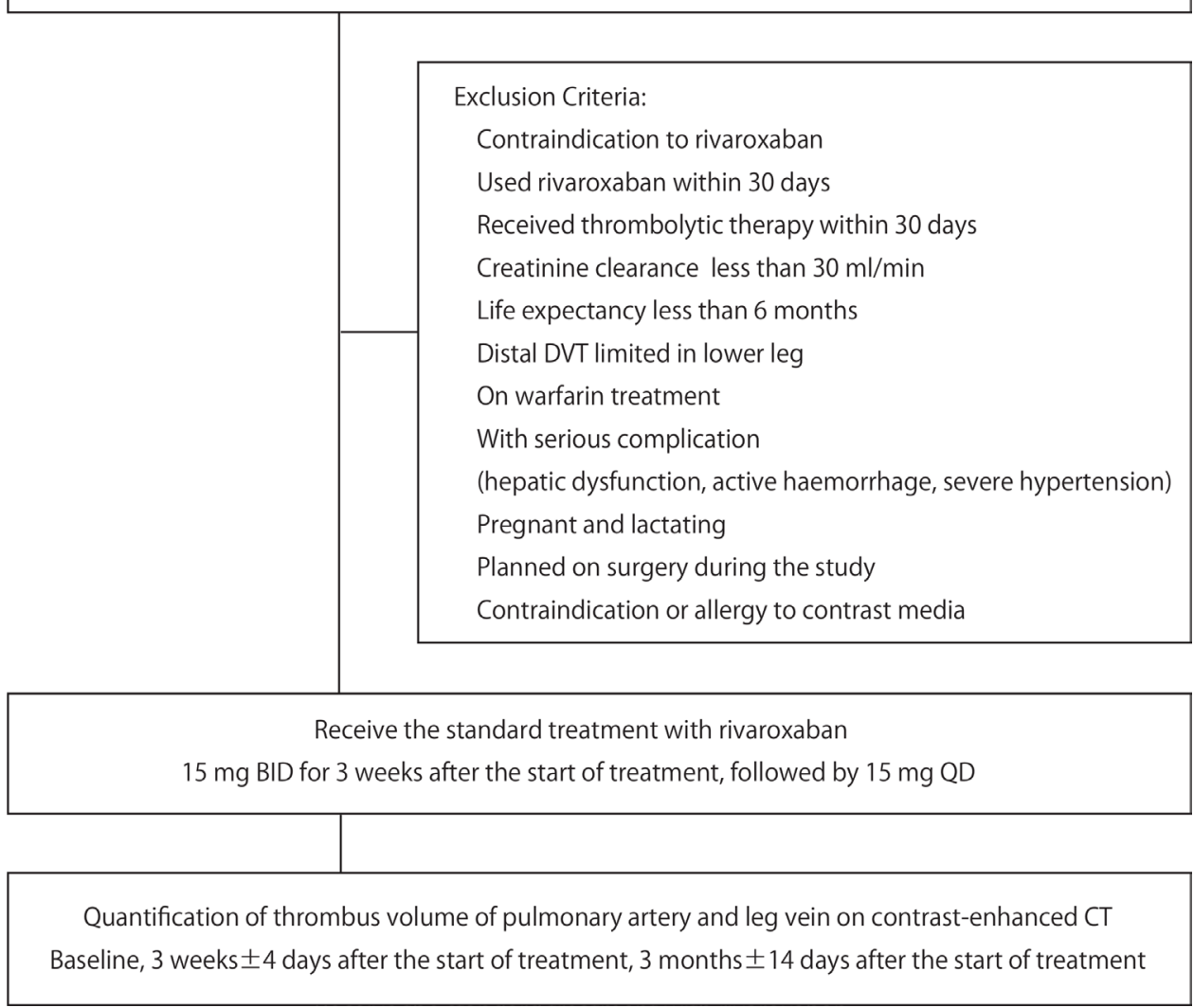

Figure 1 Flow chart of the study. DVT, deep vein thrombosis; VTE, venous thromboembolism. 


\section{METHODS AND ANALYSIS}

\section{Study design}

This is a single-arm, open-label, prospective interventional study. Figure 1 shows a flow chart of the study.

\section{Endpoints}

The primary endpoint of the study is to assess the clot regression ratio as evaluated by contrast-enhanced CT imaging. CT imaging is obtained at baseline, $21 \pm 4$ and $90 \pm 14$ days after the start of treatment with rivaroxaban. The ratio of clot volume regression is calculated at 3 weeks and 3 months after the start of treatment by comparing the clot volume to baseline. Secondary endpoints include the recurrence of symptomatic VTE and haemorrhagic complications. Recurrence of PE is defined as a new defect in pulmonary angiography, CT or lung perfusion scintigraphy with normal ventilation or a new PE confirmed at autopsy. Recurrence of DVT is defined as new thrombi or an increase in thrombus diameter of $4 \mathrm{~mm}$ or more by echography, or a new contrast defect in contrastenhanced CT. Major bleeding is defined using the International Society on Thrombosis and Hemostasis (ISTH) guideline as fatal bleeding, and/or symptomatic bleeding in a critical area or organ, such as intracranial, retroperitoneal and/or bleeding causing a fall in haemoglobin level of $20 \mathrm{~g} / \mathrm{L}$ or more, or leading to transfusion of two or more units of whole blood or red cells. ${ }^{15}$ Non-major bleeding is defined as bleeding which do not meeting the criteria of major bleeding.

\section{Eligibility criteria}

Inclusion criteria are as follows:

1. Patients with cancer who have been diagnosed with acute VTE (symptomatic or asymptomatic; PE, proximal DVT in lower or upper extremity, and thrombus in the superior or inferior vena cava) and have active cancer. Active cancer is defined as patients who have either been diagnosed with cancer within 180 days prior to obtaining consent or patients who have been treated for cancer within 180 days. This includes new, recurrent and metastatic cancer.

2. Patients who have not been treated with anticoagulation for VTE at the time of consent.

3. Patients aged from 20 to 75 years old at the time of consent due to bleeding safety concern. ${ }^{16}$

4. Written informed consent will be obtained from the patients themselves before enrolment in the study.

Exclusion criteria are as follows:

1. Patients with contraindications to rivaroxaban.

2. Patients who have taken rivaroxaban within 30 days prior to obtaining written consent.

3. Patients who received thrombolytic therapy within 30 days prior to obtaining written consent.

4. A creatinine clearance less than $30 \mathrm{~mL} / \mathrm{min}$.

5. A life expectancy of less than 6 months.

6. Distal DVT limited to the lower leg.

7. Patients currently on anticoagulation treatment.
8. Patients with serious complications at the time of consent (hepatic dysfunction (corresponding to ChildPugh classification B or C), active haemorrhage (intracranial haemorrhage, gastrointestinal haemorrhage, etc) or systolic blood pressure greater than or equal to $180 \mathrm{~mm} \mathrm{Hg}$ or diastolic blood pressure greater than or equal to $110 \mathrm{~mm} \mathrm{Hg}$ ).

9. Women who are pregnant or lactating or who may be pregnant (ie, within 12 months of last menstruation) or men who may be pregnant with partners and cannot agree to contraception during the study period.

10. Patients who, after being included in this study, agree to participate in another interventional study during the same time frame.

11. Patients who, after being included in this study, plan on having surgery during the same time frame.

12. Patients with a contraindication or allergy to contrast media.

13. Due to other safety concerns, the investigator may determine that participation in this study is inappropriate.

\section{Treatment protocol}

Patients who meet inclusion criteria but do not meet exclusion criteria are included and are started on treatment protocol. After obtaining written consent for participating in this study and registering at the case registration centre, protocol treatment is promptly started. Patients receive the standard treatment of rivaroxaban approved in Japan ( $15 \mathrm{mg}$ two times daily for 3 weeks after the start of treatment, followed by $15 \mathrm{mg} \mathrm{QD}$, this is different from other countries especially western countries). Protocol treatment is continued for $90 \pm 14$ days after the start of rivaroxaban treatment. For patients who have discontinued treatment early, a reason should be described. We plan to complete patient registration by 31 March 2020 .

Patients' background, current treatments and data from contrast-enhanced CT examinations are collected at the time of study registration, $21 \pm 4$ days after and $90 \pm 14$ days after the start of treatment with rivaroxaban. In the patients with proximal DVT, a contrast-enhanced CT after diagnosis by ultrasound is carried out to investigate the presence of PE. At the time of that scanning, a thrombus in the lower limb is concomitantly evaluated. If subsegmental PE is detected at baseline, we include these patients and re-evaluate the thrombus at time of follow-up CT. CT data to quantify thrombus volume are collected at the Department of Cardiovascular Medicine, Kyoto Prefectural University of Medicine.

\section{Quantification of thrombus volume}

Thrombus volume is quantified using a Ziostation 2 (3D medical imaging workstation, manufactured by Ziosoft, V.2.9) and by manually tracing the intravenous clot in the contrast-enhanced CT image. All pulmonary artery thrombi and leg vein thrombi are traced to calculate the total thrombus mass volume. If there is no thrombus in the CT image, the thrombus volume is determined as zero. 
Measurement results are checked by the thrombus evaluation committee and monitored for their accuracy. It should be noted that staff measuring the thrombus and evaluating the thrombus are not informed of which subject's thrombus volume at which evaluation point and therefore kept blinded.

\section{Statistical analysis}

Because this is an exploratory study, the number of cases was determined based on the feasibility of the institution. A previous study ${ }^{17}$ has reported that at 3 weeks of rivaroxaban administration, the clot regression rate on CT assessment was $59 \%$ (SD 37\%). In the study by van Es $e t$ $a l,{ }^{17}$ subjects were not limited to cancer-bearing patients, and DVT patients were excluded. Due to our study being specific to patients with cancer and high thromboembolic risk, the average thrombolysis rate is set at 20\% (SD 37\%). The number of required patients is 38 , which corresponds to a one-sided significance level of 0.025 and a power of 0.9 . The alternative hypothesis is that the one-sided test is natural as we want to show that the thrombus has regressed rather than the thrombus has simply changed. The sample size is thus set at 40 cases to account for some analysis exclusion. The population to be analysed is defined as the full analysis set (FAS) for efficacy and the safety analysis set (SAF) for adverse events. The FAS includes all patients who received the protocol treatment, with the exclusion of those who are found to be ineligible after enrolment and those for whom any information after enrolment, such as treatment initiation, cannot be obtained. The SAF includes all patients who received at least one dose of the treatment.

The clot regression ratio is defined as the ratio of regressed thrombus volume at 3 weeks and 3 months after the start of rivaroxaban compared with thrombus volume at baseline. The clot regression rate for both 3 weeks and 3 months after the start of rivaroxaban is expressed as a percentage, and the mean and 95\% CIs for the whole population are calculated based on t-distribution. If $95 \% \mathrm{CI}$ is greater than $0 \%$, we conclude that thrombus regression is statistically significant. That is, it is identical to paired t-test for each clot regression rate. If the null hypothesis is defined as having a clot regression rate of $0 \%$, this corresponds to a test with a one-sided significance level of 0.025 without adjusting the test multiplicity to account for this being an exploratory study. The frequency, percentage and $95 \%$ CIs for the percentage of symptomatic VTE recurrence and haemorrhagic complications are calculated during the study period. The incidence and severity of all adverse events for the SAF are collected during the study period.

\section{Patient and public involvement}

This research is done without patient involvement. Patients are not invited to comment on the study design and are not consulted to assess the burden of the intervention or to develop patient-relevant outcomes or interpret the results. Patients are not invited to contribute to the writing or editing of this document for readability or accuracy. Study results will be disseminated to the participants through publicly accessible medical journals.

\section{Study timeline}

Enrolment start date: June 2018.

Enrolment end date: March 2020.

Data collection end date: July 2020.

\section{DISCUSSION}

In this study, we are investigating the thrombolytic effect of rivaroxaban in patients with cancer who develop VTE. The American College of Chest Physician guideline states that LMWH is recommended as an anticoagulation treatment for VTE patients with cancer rather than vitamin $\mathrm{K}$ antagonist or DOAC. ${ }^{5}$ However, LMWH has not yet been approved for the treatment of VTE in Japan. Recently, it has been reported that some DOACs are non-inferior with respect to VTE recurrence and major bleeding events in patients with cancer. ${ }^{910}$

For patients with VTE, clot regression is an important issue. The presence of residual thrombus leads to an increased risk of DVT recurrence. In the case of DVT recurrence, it can cause reappearance of symptoms and PTS. ${ }^{18}$ Incomplete resolution of a pulmonary artery thrombus can also lead to chronic thromboembolic pulmonary hypertension $(\mathrm{CTEPH})$. CTEPH is a progressive disease with poor prognosis, classified as group 4 pulmonary hypertension in the clinical classification of pulmonary hypertension. ${ }^{19}$ To avoid these complications, clot regression in the acute phase of VTE is essential. Patients with cancer with VTE have a risk of both thrombogenicity and haemorrhage. Because patients over 75 years old who received rivaroxaban had higher bleeding rate compared with younger patients in the ROCKET $\mathrm{AF}^{16}$ trial, we excluded patients 75 years old or older in this study due to this safety concern. ${ }^{14}$ Overall, it could greatly contribute to improvements in symptoms and prognosis of patients with cancer with VTE to examine the clot regression effects and safety of rivaroxaban in the clinical practice.

Oral rivaroxaban for the treatment of VTE has been shown to be equivalent in efficacy and safety to unfractionated heparin plus warfarin with a single drug approach. Although approved dose of rivaroxaban in Japan (15 mg two times daily for 3 weeks, followed by $15 \mathrm{mg} \mathrm{QD}$ ) differs from other countries especially Western countries, the result of the J-EINSTEIN study ${ }^{12}$ was consistent with the EINSTEN study. In regard to patients with cancer with VTE, rivaroxaban was associated with lower VTE recurrence and consistent major bleeding risk, but higher minor bleeding risk, compared with LMWH. ${ }^{10}$ However, the efficacy of the Japanese dose of rivaroxaban in patients with cancer with VTE has not been well investigated. In this study, we will examine whether the Japanese dose of rivaroxaban can be used effectively and safely for the treatment of VTE patients with cancer.

There are some limitations in this study. First, the study is exploratory with a small number of patients. To evaluate the effect of rivaroxaban on the reduction of clot burden, we must perform a comparative study. Unfortunately, we 
are unable to use a placebo due to ethical reasons. Our aim in this study is to evaluate an efficacy of rivaroxaban on clot regression in the usual clinical practice. This clot regression includes a fibrinolytic effect both of rivaroxaban and biological function. Clinically, it is not necessary to separate these fibrinolytic effects clearly. Although we have not included a placebo group, it would appear that the natural regression rate of a thrombus is small; therefore, the fibrinolytic effect of rivaroxaban can be evaluated. Moreover, we do not intend to investigate the potential superiority of rivaroxaban over other anticoagulation agents. Second, as we have not strictly defined thrombus type as inclusion criteria, thrombi in different patients will inevitably vary in size and location. Finally, this study does not have sufficient sample size to evaluate secondary endpoints such as recurrence of symptomatic VTE or haemorrhagic complications. Despite these limitations, this study seems to greatly contribute to improving symptoms and prognosis in patients with cancer with VTE.

\section{ETHICS AND DISSEMINATION}

This study is based on the ethical principles based on the Declaration of Helsinki and its revised version. In order to receive continuous review by the ethics review committee, the research manager conducts a yearly overview of the study's status and reports in writing to the head of the organisation. Before participating in the study, the investigator informs subjects using the explanatory document and provides enough time to ask questions to make an informed decision. After confirming that the subject fully understands the content, written consent is obtained from the patient for participation in the study. The parties concerned with this study take full consideration of the subject's personal information and privacy protection in accordance with relevant laws and regulations. In addition, the information obtained in this study will not be provided to third parties, except when requested by a public organisation. Study results will be disseminated through peer-reviewed journals.

\section{Author affiliations \\ ${ }^{1}$ Cardiovascular Medicine, Graduate School of Medical Science, Kyoto Prefectural University of Medicine, Kyoto, Japan \\ ${ }^{2}$ Biostatistics, Graduate School of Medicine, Hokkaido University, Sapporo, Japan ${ }^{3}$ Medical Innovation and Translational Medical Science, Graduate School of Medical Science, Kyoto Prefectural University of Medicine, Kyoto, Japan \\ ${ }^{4}$ The Center for Quality Assurance in Research and Development, Kyoto Prefectural University of Medicine, Kyoto, Japan \\ ${ }^{5}$ Cardiovascular Medicine, Kyoto City Hospital, Kyoto, Japan \\ ${ }^{6}$ Cardiovascular Medicine, Kyoto First Red Cross Hospital, Kyoto, Japan \\ ${ }^{7}$ Cardiovascular Medicine, Kyoto Second Red Cross Hospital, Kyoto, Japan}

Contributors NN wrote the draft manuscript. ST, NN, IY, KI, AY and SM planned and organised the study. TK, TO, TS and HF conducted the study. SM designed and directed the study. All authors read and approved the manuscript.

Funding This study is conducted using normal insurance practices. Based on the contract for the investigator-initiated clinical trial, the research manager conducted a study commissioned by Bayer Yakuhin. Bayer Yakuhin give funding for consignment expenses, such as data management, monitoring and statistical analysis.

Competing interests None declared.

Patient consent for publication Not required.
Ethics approval This study was approved by the institutional review board of the Kyoto Prefectural University of Medicine (ERB-C-891-2).

Provenance and peer review Not commissioned; externally peer reviewed.

Open access This is an open access article distributed in accordance with the Creative Commons Attribution Non Commercial (CC BY-NC 4.0) license, which permits others to distribute, remix, adapt, build upon this work non-commercially, and license their derivative works on different terms, provided the original work is properly cited, appropriate credit is given, any changes made indicated, and the use is non-commercial. See: http://creativecommons.org/licenses/by-nc/4.0/.

\section{REFERENCES}

1 Büller HR, Davidson BL, Decousus H, et al. Fondaparinux or enoxaparin for the initial treatment of symptomatic deep venous thrombosis: a randomized trial. Ann Intern Med 2004;140:867-73.

2 Büller HR, Davidson BL, Decousus H, et al. Subcutaneous fondaparinux versus intravenous unfractionated heparin in the initial treatment of pulmonary embolism. $N$ Engl J Med 2003;349:1695-702.

3 Buller HR, Cohen AT, Davidson B, et al. Idraparinux versus standard therapy for venous thromboembolic disease. $N$ Engl J Med 2007;357:1094-104.

4 Posch F, Königsbrügge O, Zielinski C, et al. Treatment of venous thromboembolism in patients with cancer: a network meta-analysis comparing efficacy and safety of anticoagulants. Thromb Res 2015;136:582-9.

5 Kearon C, Akl EA, Ornelas J, et al. Antithrombotic therapy for VTE disease: chest guideline and expert panel report. Chest 2016;149:315-52.

6 Bauersachs R, Berkowitz SD, Brenner B, et al. Oral rivaroxaban for symptomatic venous thromboembolism. N Engl J Med 2010;363:2499-510.

7 Büller HR, Prins MH, Lensin AWA, et al. Oral rivaroxaban for the treatment of symptomatic pulmonary embolism. $N$ Engl J Med 2012;366:1287-97.

8 Prins MH, Lensing AWA, Brighton TA, et al. Oral rivaroxaban versus enoxaparin with vitamin $\mathrm{K}$ antagonist for the treatment of symptomatic venous thromboembolism in patients with cancer (EINSTEIN-DVT and EINSTEIN-PE): a pooled subgroup analysis of two randomised controlled trials. Lancet Haematol 2014;1:e37-46.

9 Raskob GE, van Es N, Verhamme P, et al. Edoxaban for the treatment of cancer-associated venous thromboembolism. N Engl J Med 2018;378:615-24.

10 Young AM, Marshall A, Thirlwall J, et al. Comparison of an oral factor Xa inhibitor with low molecular weight heparin in patients with cancer with venous thromboembolism: results of a randomized trial (SELECT-D). J Clin Oncol 2018;36:2017-23.

11 Carrier M, Abou-Nassar K, Mallick R, et al. Apixaban to prevent venous thromboembolism in patients with cancer. $N$ Engl J Med 2019;380:711-9.

12 Khorana AA, Soff GA, Kakkar AK, et al. Rivaroxaban for thromboprophylaxis in high-risk ambulatory patients with cancer. $N$ Engl J Med 2019;380:720-8.

13 Yamada N, Hirayama A, Maeda $\mathrm{H}$, et al. Oral rivaroxaban for Japanese patients with symptomatic venous thromboembolism - the J-EINSTEIN DVT and PE program. Thromb J 2015;13:2.

14 Antignani PL, Allegra C, Fareed J. Treatment of deep vein thrombosis with rivaroxaban and its potential to prevent the post-thrombotic syndrome. Int Angiol 2019;38:17-21.

15 Schulman S, Kearon C, Subcommittee on Control of Anticoagulation of the Scientific and Standardization Committee of the International Society on Thrombosis and Haemostasis. Definition of major bleeding in clinical investigations of antihemostatic medicinal products in non-surgical patients. J Thromb Haemost 2005;3:692-4.

16 Goodman SG, Wojdyla DM, Piccini JP, et al. Factors associated with major bleeding events: insights from the rocket AF trial (rivaroxaban once-daily oral direct factor Xa inhibition compared with vitamin $\mathrm{K}$ antagonism for prevention of stroke and embolism trial in atrial fibrillation). J Am Coll Cardiol 2014;63:891-900.

17 van Es J, Douma RA, Kamphuisen PW, et al. Clot resolution after 3 weeks of anticoagulant treatment for pulmonary embolism: comparison of computed tomography and perfusion scintigraphy. $J$ Thromb Haemost 2013;11:679-85.

18 Prandoni P, Lensing AW, Cogo A, et al. The long-term clinical course of acute deep venous thrombosis. Ann Intern Med 1996;125:1-7.

19 Simonneau G, Montani D, Celermajer DS, et al. Haemodynamic definitions and updated clinical classification of pulmonary hypertension. Eur Respir J 2019;53. 